\title{
Enhancing chemosensitivity in oral squamous cell carcinoma by lentivirus vector-mediated RNA interference targeting EGFR and MRP2
}

\author{
YING-JU CHEN $^{1}$, SHIUAN-YIN CHEN ${ }^{1}$, RONALD LOVEL ${ }^{1}$, YI-CHU KU ${ }^{1}$, YI-HUI LAI ${ }^{1}$, \\ CHIAO-LING HUNG ${ }^{1}$, YU-FEN LI ${ }^{2}$, YIN-CHE LU ${ }^{1,3,4}$ and CHIEN-KUO TAI ${ }^{1}$
}

\begin{abstract}
${ }^{1}$ Department of Life Science and Institutes of Molecular Biology and Biomedical Science, National Chung Cheng University, Chia-Yi 62102; ${ }^{2}$ Institute of Biostatistics, China Medical University, Taichung 40402; ${ }^{3}$ Division of Hematology-Oncology, Ditmanson Medical Foundation Chia-Yi Christian Hospital, Chia-Yi 60002; ${ }^{4}$ Department of Health and Nutrition, Chia Nan University of Pharmacy and Science, Tainan 71710, Taiwan, R.O.C.
\end{abstract}

Received December 4, 2014; Accepted January 14, 2016

DOI: $10.3892 / 01.2016 .4883$

\begin{abstract}
Oral cancer is the eighth most common type of cancer among men worldwide, with an age-standardized rate of 6.3 per 100,000 , and is the fourth leading cause of cancer-associated mortality among men in Taiwan. Cisplatin and 5-fluorouracil (5-FU) are two of the most frequently utilized chemotherapy drugs for the treatment of oral cancer. Although oral cancer patients initially benefit from chemotherapy with these drugs, they may develop resistance to them, which worsens their prognosis and reduces survival rates. It has been reported that increased levels of epidermal growth factor receptor (EGFR) and multidrug resistance-associated protein 2 (MRP2) induce drug resistance in numerous types of human cancer. Therefore, the present study employed lentivirus vector-mediated RNA interference (RNAi) in order to target the genes encoding EGFR and MRP2 in the oral squamous cell carcinoma cell line OC2. It was observed that RNAi-mediated downregulation of EGFR or MRP2 increased the sensitivity to 5-FU and cisplatin in OC2 cells. Downregulation of EGFR resulted in significant suppression of OC2 tumor growth following 5-FU administration. However, simultaneous downregulation of the two genes did
\end{abstract}

Correspondence to: Professor Chien-Kuo Tai, Department of Life Science and Institutes of Molecular Biology and Biomedical Science, National Chung Cheng University, 168 University Road, Min-Hsiung, Chia-Yi 62102, Taiwan, R.O.C.

E-mail: biockt@ccu.edu.tw

Dr Yin-Che Lu, Department of Health and Nutrition, Chia Nan University of Pharmacy and Science, No. 60, Section 1, Erren Road, Tainan 71710, Taiwan, R.O.C.

E-mail: 04688@cych.org.tw

Key words: oral cancer, chemosensitivity, RNA interference, epidermal growth factor receptor, multidrug resistance-associated protein 2 not further suppress the tumor growth, indicating that MRP2 does not have a significant role in the chemosensitivity of EGFR-downregulated cells to 5-FU. In contrast, downregulation of $M R P 2$ was demonstrated to significantly enhance the therapeutic effects of cisplatin in EGFR-downregulated OC2 tumors. The observation that the expression of $M R P 2$ was positively correlated with the level of cisplatin resistance in cells suggests that RNAi-mediated downregulation of MRP2 may be applicable as a therapeutic approach toward reversing MRP2-dependent cisplatin resistance in oral cancer.

\section{Introduction}

Oral cancer is the eighth most common type of cancer among men worldwide, with an age-standardized rate of 6.3 per $100,000(1,2)$, and is the fourth leading cause of cancer-associated mortality among men in Taiwan (3). In addition, oral cancer has been ranked highest with respect to the rates of incidence and mortality among men aged 25-44 years in Taiwan since 2005 (3). Chemotherapy has been used as the primary treatment for patients exhibiting recurrent or metastatic cancers, including oral cancer (4). Of the various chemotherapy drugs available, cisplatin and 5-fluorouracil (5-FU) are the two most frequently utilized for the treatment of oral cancer (5). However, the efficacy of cancer chemotherapy is often limited by the development of drug resistance by the cancer cells in clinical practice (6).

It has been demonstrated that multidrug resistance results largely from the expression of adenosine triphosphate-binding cassette (ABC) transporters with broad drug specificity (7-9). Multidrug resistance-associated protein 2 (MRP2), a member of the ABC transporter superfamily, has been observed in various tumors to confer resistance to a number of therapeutic drugs, including cisplatin (10-12). The levels of MRP2 messenger (m)RNA in certain human cancer cells are inversely correlated with their sensitivity to cisplatin $(13,14)$. Thus, increased expression of the gene encoding MRP2 in cancer cells may reduce the intracellular accumulation of cisplatin (15). By contrast, a reduction in MRP2 expression 
using the RNA interference (RNAi) approach was demonstrated to reverse MRP2-dependent cisplatin resistance in human ovarian carcinoma (16) and nasopharyngeal carcinoma cells (17). In addition, it has been confirmed that the mRNA levels of $M R P 2$ are markedly correlated with the half-maximal inhibitory concentration $\left(\mathrm{IC}_{50}\right)$ value of 5-FU in esophageal carcinoma cells (18). Therefore, knockdown of MRP2 may influence the sensitivity of cancer cells to 5-FU.

Epidermal growth factor receptor (EGFR), which is one of most well-studied receptor tyrosine kinases, promotes cancer cell growth and is associated with drug resistance (19). Thus, it has emerged as a significant target for the development of anticancer therapy (20). Although cancer patients may initially benefit from EGFR-targeted therapies, drug resistance frequently develops due to crosstalk or inappropriate activation of downstream signaling pathways of EGFR (21). Instead of using EGFR-targeted monoclonal antibodies or small-molecule inhibitors to inhibit the activity of EGFR, the expression of its gene, EGFR, in cancer cells may be silenced using RNAi techniques (22). Therefore, lentivirus vector-mediated RNAi was used in the present study to silence the expression of EGFR and MRP2 in oral cancer. The aim of the present study was to enhance the chemosensitivity of oral cancer cells to the chemotherapeutic drugs cisplatin and 5-FU, and to reverse the drug resistance exhibited by these cells.

\section{Materials and methods}

Cell cultures. The oral squamous cell carcinoma (OSCC) cell lines OC2 and OCSL $(23,24)$, derived from two Taiwanese male patients who demonstrated habits of alcohol drinking, betel quid chewing and cigarette smoking, were maintained in RPMI 1640 medium (catalog no., 11875-085; Gibco; Thermo Fisher Scientific, Inc., Waltham, MA, USA) supplemented with $10 \%$ fetal bovine serum (FBS; catalog no., 10437-028; Invitrogen; Thermo Fisher Scientific, Inc.). The OSCC cell lines SCC25 [obtained from American Type Culture Collection (ATCC), Manassas, VA, USA] and HSC3 (obtained from Health Science Research Resources Bank, Osaka, Japan) were maintained in Dulbecco's modified Eagle's medium (DMEM)/Ham's Nutrient Mixture F-12 (1:1 mixture; catalog no., 11330-032, Gibco; Thermo Fisher Scientific, Inc.) supplemented with $10 \%$ FBS. SCC25 cells were additionally supplied with $400 \mathrm{ng} / \mathrm{ml}$ hydrocortisone (Sigma-Aldrich, St. Louis, MO, USA). The transformed human embryonic kidney cell line 293T (obtained from ATCC) was maintained in DMEM with $10 \%$ FBS.

Reverse transcription-polymerase chain reaction (RT-PCR) analysis. Total RNA was extracted from cells using REzol ${ }^{\mathrm{TM}}$ C \& T reagent (catalog no., KP200CT; Protech Technology Enterprise Co., Ltd., Taipei, Taiwan), according to the manufacturer's protocol. Complementary DNA (cDNA) was synthesized from aliquots of RNA using SuperScript ${ }^{\circledR}$ III First-Strand Synthesis System (catalog no., 18080-051; Thermo Fisher Scientific, Inc.) according to the manufacturer's protocol. DNase I (catalog no., 18068-015; Thermo Fisher Scientific, Inc.) included in this system was used to treat the RNA sample to eliminate DNA. RT-PCR was performed using Taq DNA Polymerase Master Mix Red (catalog no., A180301;
Ampliqon, Odense M, Denmark) and MyCycler ${ }^{\mathrm{TM}}$ Thermal Cycler (catalog no., 170-9703; Bio-Rad Laboratories, Inc., Hercules, CA, USA). EGFR and MRP2 cDNA was amplified using the following primers (Protech Technology Enterprise Co., Ltd.): EGFR forward, 5'-CCAAACAATTAGCCTGGA CA-3' and reverse, 5'-CGCGACCCTTAGGTATTCTG-3'; and MRP2 forward, 5'-TGCAGCCTCCATAACCATGAG-3' and reverse, 5'-GATGCCTGCCATTGGACCTA-3'. Glyceraldehyde 3-phosphate dehydrogenase (GAPDH) cDNA was additionally amplified as an internal control with primers forward, 5'-GCCATCAATGACCCCTTAATT-3' and reverse, 5'-TTGACGGTGCCATGGAATTT-3'. The PCR programs were set to perform the following steps: For $E G F R, 95^{\circ} \mathrm{C}$ for $5 \mathrm{~min}$, followed by 30 cycles of successive incubation at $95^{\circ} \mathrm{C}$ for $30 \mathrm{sec}, 54^{\circ} \mathrm{C}$ for $30 \mathrm{sec}$ and $72^{\circ} \mathrm{C}$ for $30 \mathrm{sec}$; for $M R P 2,95^{\circ} \mathrm{C}$ for $5 \mathrm{~min}$, followed by 38 cycles of successive incubation at $95^{\circ} \mathrm{C}$ for $30 \mathrm{sec}, 63^{\circ} \mathrm{C}$ for $30 \mathrm{sec}$ and $72^{\circ} \mathrm{C}$ for $30 \mathrm{sec}$; for $\mathrm{GAPDH}, 95^{\circ} \mathrm{C}$ for $5 \mathrm{~min}$, followed by 26 cycles of successive incubation at $95^{\circ} \mathrm{C}$ for $30 \mathrm{sec}, 54^{\circ} \mathrm{C}$ for $30 \mathrm{sec}$ and $72^{\circ} \mathrm{C}$ for $30 \mathrm{sec}$. The PCR products and DNA ladder (catalog no., 02001-500; OmicsBio, Taipei, Taiwan) were resolved on a $2 \%$ agarose gel (catalog no., 0710-250G; Amresco, Solon, $\mathrm{OH}$, USA) and visualized by ethidium bromide (catalog no., X328; Protech Technology Enterprise Co., Ltd.) staining. Expression of EGFR and MRP2 was quantified using ImageJ software v1.49 (http://imagej.nih.gov/ij/download.html; accessed October 21, 2014) and normalized against that of GAPDH.

Lentivirus vector production and transfection. Lentivirus vectors were produced by transient transfection of pCMVdeltaR8.91,pMD.G and pLKO.1-puro or pLKO-TRC008 vectors, carrying a short-hairpin RNA (shRNA), into 293T cells using calcium phosphate precipitation $\left[2.5 \mathrm{M} \mathrm{CaCl}_{2}\right.$, catalog no., 1332-01 (J.T. Baker, Phillipsburg, NJ, USA); $50 \mathrm{mM}$ 4-(2-hydroxyethyl)-1-piperazineethanesulfonic acid (HEPES), catalog no., 15630-080 (Gibco; Thermo Fisher Scientific, Inc.); 2X HEPES-buffered saline, catalog no., 7021 (Sigma-Aldrich); 10 mM KCl, catalog no., 3040-01 (J.T. Baker); 1.4 mM $\mathrm{Na}_{2} \mathrm{HPO}_{4}$, catalog no., 0404-500G (Amresco); $240 \mathrm{mM} \mathrm{NaCl}$, catalog no., 0241-2.5KG (Amresco); $10 \mathrm{mM}$ chloroquine, catalog no., c6628 (Sigma-Aldrich)], as described previously (25). pLKO plasmids were obtained from the National RNAi Core Facility at the Academia Sinica in Taipei, Taiwan. The shRNA sequences specific for $E G F R$ and $M R P 2$ were 5'-GCTGGATGATAGACGCAGATA-3' and 5'-GCCGGT GGTCAGATTATCATT-3', respectively. OSCC cells infected with shRNA-expressing lentivirus vectors (shEGFR or shMRP2) were selected by incubation with $2 \mu \mathrm{g} / \mathrm{ml}$ puromycin (Sigma-Aldrich) or $20 \mu \mathrm{g} / \mathrm{ml}$ blasticidin (Sigma-Aldrich) for 2 days. Cells infected with sh-red fluorescent protein (RFP) were used as a control.

Western blot analysis. Cells were lysed by the treatment of RIPA Lysis and Extraction Buffer (catalog no., 89900; Thermo Fisher Scientific, Inc.) according to the manufacturer's protocol. The cell lysates were resuspended in sodium dodecyl sulfate (SDS) gel-loading buffer [62.5 mM Tris- $\mathrm{HCl}(\mathrm{pH}$ 6.8); $2 \%$ SDS; $10 \%$ glycerol; $100 \mathrm{mM}$ dithiothreitol; $0.01 \%$ bromophenol blue) and boiled for $5 \mathrm{~min}$. Protein samples were run on SDS-10\% polyacrylamide gels with protein marker 
(catalog no., PT-PS03; Protech Technology Enterprise Co., Ltd.) at 120 volts for $90 \mathrm{~min}$ and transferred onto polyvinylidene difluoride membranes (catalog no., 162-0177, Bio-Rad Laboratories, Inc.) at 90 volts for $80 \mathrm{~min}$. The membranes were blocked for $2 \mathrm{~h}$ at room temperature in TBST [100 mM Tris- $\mathrm{HCl}$ (pH 7.5); $150 \mathrm{mM} \mathrm{NaCl} ; 0.1 \%$ Tween 20] with 5\% non-fat dry milk (catalog no., 1706404, Bio-Rad Laboratories, Inc.), and probed with rabbit polyclonal anti-EGFR antibody (dilution, 1:1,000; catalog no., ab2430; Abcam, Cambridge, MA, USA) or mouse monoclonal anti- $\beta$-actin antibody (dilution, 1:5,000; catalog no., A5316; Sigma-Aldrich). The specific protein signals were detected by using an enhanced chemiluminescence kit (catalog no., WBKLS0500; Merck Millipore, Darmstadt, Germany).

Cell proliferation analysis by 3-(4,5-dimethylthiazol-2-yl)-5(3-carboxymethoxyphenyl)-2-(4-sulfophenyl)-2H-tetrazolium (MTS) assay. To determine the proliferation rate of cells transfected with shEGFR, the cells were seeded onto replicate 96-well plates (catalog no., 30096; SPL Life Sciences, Co., Ltd., Gyeonggi, Korea) at a density of 400 cells/well. Following overnight culture, cell proliferation was determined daily by MTS assay, using the CellTiter $96^{\circledR}$ AQueous One Solution Cell Proliferation Assay kit (Promega Corporation, Madison, WI, USA).

Colony formation assay. A total of 400 cells were seeded onto 6-well plates (catalog no., 30006; SPL Life Sciences, Co., Ltd.) and cultured for 1-2 weeks or until colonies of cells were visible with the naked eye. Cell colonies were stained with $0.4 \%$ crystal violet (Sigma-Aldrich) in $50 \%$ methanol (catalog no., 322415; Sigma-Aldrich) for $10 \mathrm{~min}$, followed by washing with phosphate-buffered saline (PBS), air-drying at room temperature for 2 days and counting with the naked eye. Experiments were performed in triplicate.

Determination of the $I C_{50}$ values of drugs. To determine the $\mathrm{IC}_{50}$ values of 5-FU (catalog no., F6627; Sigma-Aldrich) and cisplatin (catalog no., P4394; Sigma-Aldrich), cells were seeded onto replicate 96-well plates at a density of 2,000 cells/well. Following overnight culture, the cells were incubated with 5 -FU or cisplatin at various concentrations $(0.00,0.25,0.50,1$, $2,4,6,8,10,12,14$ and $16 \mu \mathrm{g} / \mathrm{ml}$ ) for 3 days, and the remaining viable cells were determined by MTS assay.

Apoptosis analysis. OC2 and OC2 cisplatin-resistant (CisR) cells were exposed to increasing concentrations of cisplatin, ranging from $1-16 \mu \mathrm{g} / \mathrm{ml}$ for $24 \mathrm{~h}$. The cells were subsequently harvested with trypsin (catalog no., 25200-072; Gibco; Thermo Fisher Scientific, Inc.), washed with PBS and resuspended in binding buffer (catalog no., AVF250, Strong Biotech Corp., Taipei, Taiwan) containing the optimal concentration of calcium required for Annexin $\mathrm{V}$ to bind to phosphotidylserine on the cell surface. Annexin V-fluorescein isothiocyanate (Strong Biotech Corp.) and propidium iodide (PI; Sigma-Aldrich) were added to the cell suspension and allowed to incubate in the dark for $15 \mathrm{~min}$ at room temperature. The cells were subsequently analyzed by flow cytometry using a FACSCalibur ${ }^{\mathrm{TM}}$ with BD CellQuest ${ }^{\mathrm{TM}}$ Pro Software version 6.0 (BD Biosciences, San Jose, CA, USA), and the percentages of early (Annexin $\mathrm{V}^{+}, \mathrm{PI}^{-}$) and late apoptotic cells (Annexin $\mathrm{V}^{+}, \mathrm{PI}^{+}$) were calculated according to the manufacturer's instructions.

Development of the OC2CisR cell line. OC2 cells were seeded onto 6-cm culture plates (catalog no., 20101, SPL Life Sciences, Co., Ltd.) at $50 \%$ confluency. Following overnight culture, the cells were incubated with cisplatin at a concentration of $0.10 \mu \mathrm{g} / \mathrm{ml}$, until the cultures approached confluency. The cells were subcultured onto another $6-\mathrm{cm}$ plate at $50 \%$ confluency and incubated with cisplatin at a concentration of $0.25 \mu \mathrm{g} / \mathrm{ml}$. Following the repetition of the procedure with the increased concentrations of 0.50 and $0.75 \mu \mathrm{g} / \mathrm{ml}$, the cells were able to survive in a medium containing $0.75 \mu \mathrm{g} / \mathrm{ml}$ cisplatin and the cells were designated as the OC2CisR cell line.

In vivo experiments. OSCC cells were implanted into the right dorsal flank of athymic female BALB/c nude mice (randomly divided into groups, n=6/group; National Laboratory Animal Center, Taipei, Taiwan). When the tumor volumes reached $\sim 100 \mathrm{~mm}^{3}$, the mice were administered intraperitoneal injections of 5 -FU (15 mg/kg/day) for a total of 6 treatments, or cisplatin at a weekly dose of $3 \mathrm{mg} / \mathrm{kg}$ for a total of 3 treatments. The tumor volumes were measured daily, and the maximum allowable size of the tumors was $15 \mathrm{~mm}$ in diameter. The housing conditions of the mice were as follows: 12-h light/12-h dark cycle; temperatures of $18-23^{\circ} \mathrm{C}$ with $40-60 \%$ humidity; and water and food accessible at all times. All experiments were performed under license from the Institutional Animal Care and Use Committee of the National Chung Cheng University.

Statistical analysis. Student's $t$-tests were performed for statistical analysis of relative gene expression, cell proliferation, cell apoptosis, colony-formation, $\mathrm{IC}_{50}$ value and relative tumor volume. All data are presented as the mean \pm standard error (SE). For the comparison of tumor growth rates, generalized linear models with generalized estimated equations were used to account for the correlation between the repeated measurements. A significant interaction between time and group in the generalized linear models meant that the growth rates of the groups were changing over time in various ways. A P-value of $<0.05$ was considered to indicate a statistically significant difference. Analyses were performed using SAS version 9.3 (SAS Institute Inc., Cary, NC, USA).

\section{Results}

Expression levels of EGFR and MRP2 in OSCC cell lines. RT-PCR was employed to examine the expression of $E G F R$ and MRP2 in the OSCC cell lines OC2, OCSL, SCC25 and HSC3. Fig. 1 shows the differential expression of these two genes among the four cell types. With respect to the two OSCC cell types derived from Taiwanese male patients who habitually drank alcohol, chewed betel-quid and smoked cigarettes, namely, OC2 and OCSL, the former exhibited increased expression of EGFR and $M R P 2$ compared with the the latter $(\mathrm{P}<0.05)$. Thus, OC2 was selected as the target cell line for evaluation of RNAi-mediated downregulation of EGFR and MRP2. 


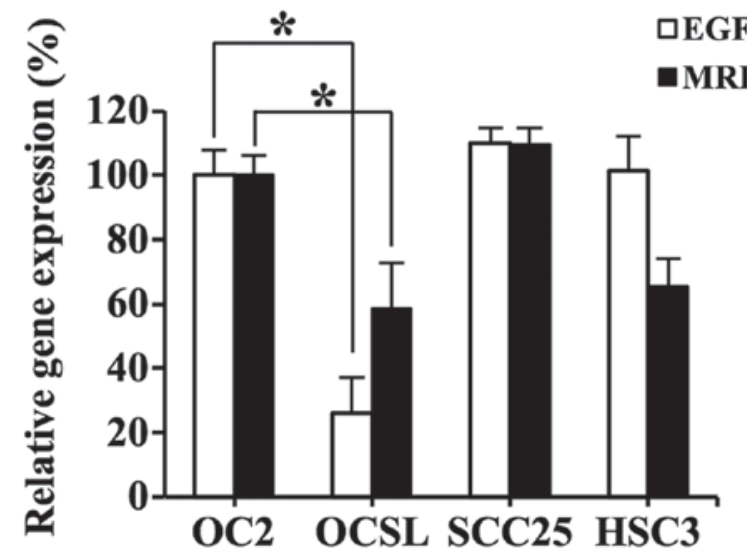

Figure 1. Relative expression levels of EGFR and $M R P 2$ in OSCC cell lines. The messenger RNA levels of EGFR and MRP2 in OSCC cell lines were quantified using reverse transcription-polymerase chain reaction. The expression levels of EGFR and MRP2 in OC2 cells were assigned a value of $100 \%$ as controls. Data are presented as the mean \pm standard error. ${ }^{*} \mathrm{P}<0.05$. EGFR, epidermal growth factor receptor; MRP2, multidrug resistance-associated protein 2; OSCC, oral squamous cell carcinoma.

Downregulation of EGFR expression inhibits the growth of OC2 cells. Western blot analysis was utilized to determine the expression of EGFR protein in OC2 cells transfected with shEGFR, thus confirming the specificity of RNAi-mediated silencing of EGFR. It was observed that shEGFR transfection effectively reduced the expression of EGFR protein in OC2 cells (Fig. 2A). In addition, MTS and colony formation assays were employed to investigate the proliferation rate and colony-forming ability, respectively, of the transfected OC2 cells. A significant difference was observed between the growth of shEGFR- and shRFP-transfected OC2 cells $(\mathrm{P}<0.01$; Fig. 2B and $\mathrm{C})$, indicating that downregulation of $E G F R$ expression inhibited the growth of the $\mathrm{OC} 2$ cells.

Downregulation of EGFR and MRP2 enhances the sensitivity of OC2 cells to 5-FU.OC2 cells transfected with shMRP2 alone or in combination with shEGFR were established to determine whether $M R P 2$ downregulation enhanced the chemosensitivity of OC2 cells to 5-FU. Fig. 3A shows the reduced expression of $E G F R$ and MRP2 in OC2/shEGFR/shMRP2 cells compared with the control group OC2/shRFP $(\mathrm{P}<0.01)$. Transfection of OC2 cells with shEGFR was effective at sensitizing the cells to $5-\mathrm{FU}$ according to the $\mathrm{IC}_{50}$ value, which was determined using the MTS assay (Fig. 3B). In addition, downregulation of $M R P 2$ increased the sensitivity of the cells to 5-FU. However, knockdown of MPR2 and EGFR did not further decrease the $\mathrm{IC}_{50}$ value of 5-FU, compared with the cells transfected with shEGFR alone.

Mice bearing subcutaneous OC2 tumors were treated with 5-FU to determine whether the RNAi-induced downregulation of EGFR and MRP2 expression confers a therapeutic benefit in vivo (Fig. 3C). Downregulation of EGFR resulted in a significant suppression of tumor growth following 5-FU administration, compared with the control group (OC2/shRFP; Fig. 3D). However, downregulation of $M R P 2$ and $E G F R$ did not further suppress tumor growth, indicating that MRP2 does not have a significant role in the chemosensitivity of EGFR-downregulated OC2 cells to 5-FU.
A

EGFR

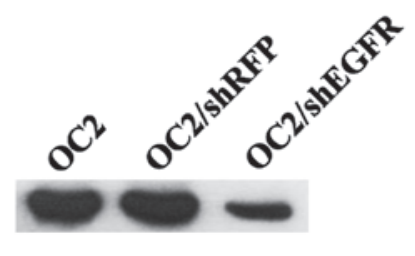

$\beta$-actin

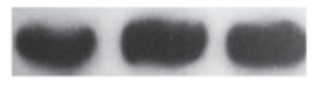

B

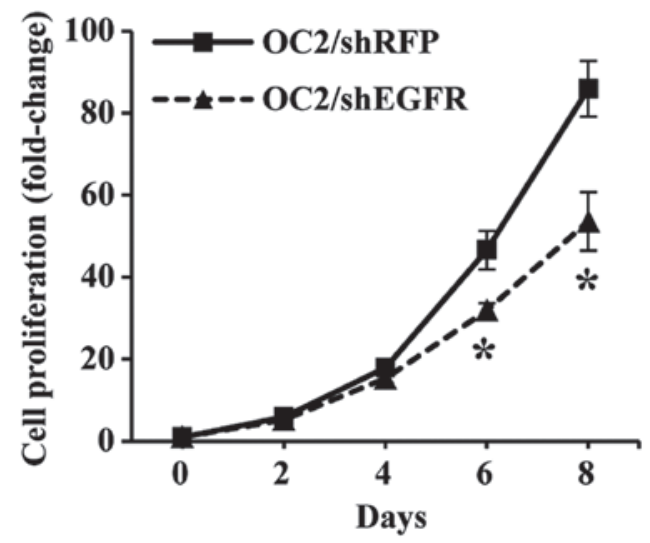

C

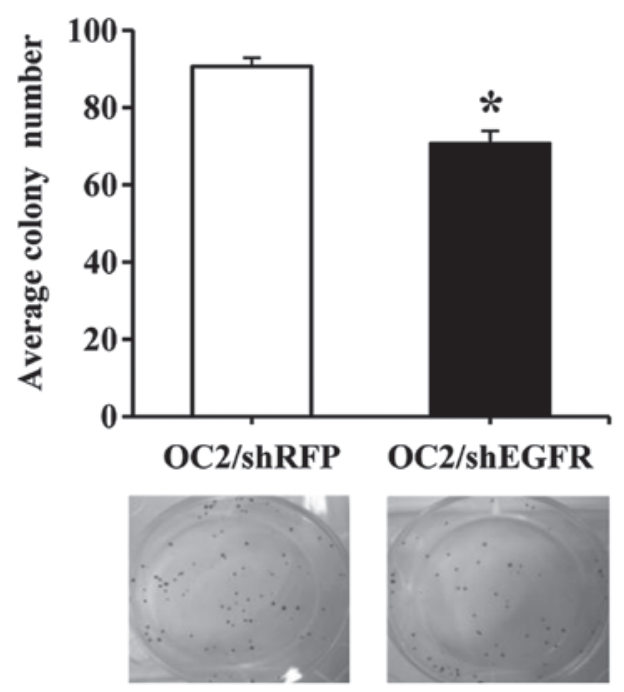

Figure 2. Downregulation of EGFR expression inhibits the growth of OC2 cells. (A) Western blot analysis of EGFR protein (170 kDa) expression in OC2 cells transfected with shEGFR or shRFP. $\beta$-actin $(42 \mathrm{kDa})$ was used as a loading control. (B) The effect of EGFR downregulation on the proliferation of OC2 cells was determined by 3-(4,5-dimethylthiazol-2-yl)-5-(3-car boxymethoxyphenyl)-2-(4-sulfophenyl)-2H-tetrazolium assay. The increase in cell populations was compared with the number of cells on day 0 , which was assigned a value of 1 . A significant difference was observed between the proliferation of shEGFR- and shRFP-transfected OC2 cells. (C) Quantitative analysis of the number of colonies formed using the colony formation assay. A significant difference was observed between the colony-forming ability of shEGFR- and shRFP-transfected OC2 cells. Data are presented as the mean \pm standard error. ${ }^{*} \mathrm{P}<0.01$. EGFR, epidermal growth factor receptor; sh, small hairpin; RFP, red fluorescent protein.

Development of the OC2CisR cell line. Previous studies have demonstrated that MRP2 confers resistance to cisplatin in certain types of cancer (9-11). A cisplatin-resistant OC2 cell line (designated OC2CisR) was generated for use as the target cell for subsequent experiments, in order to determine 
A

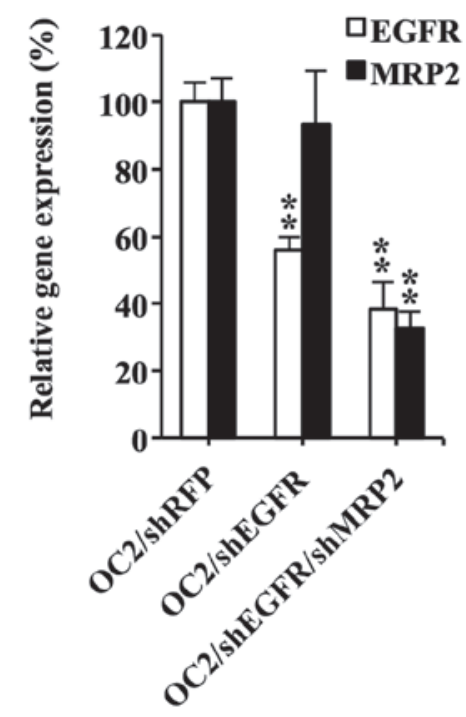

C

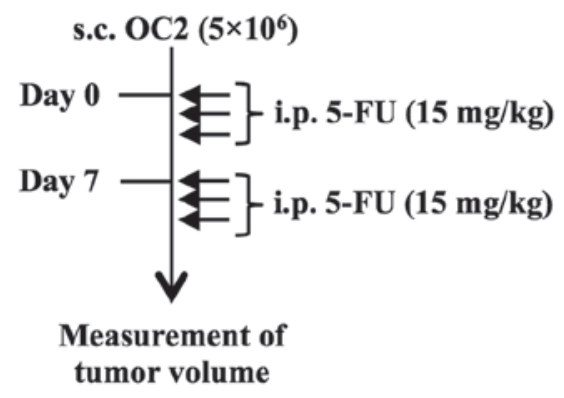

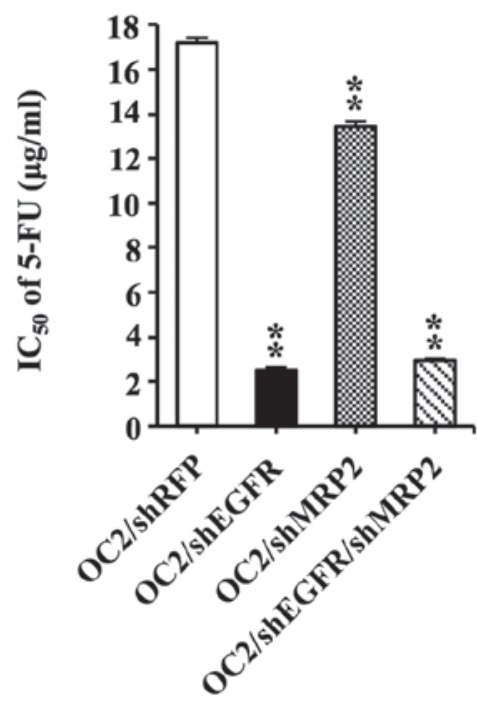

D

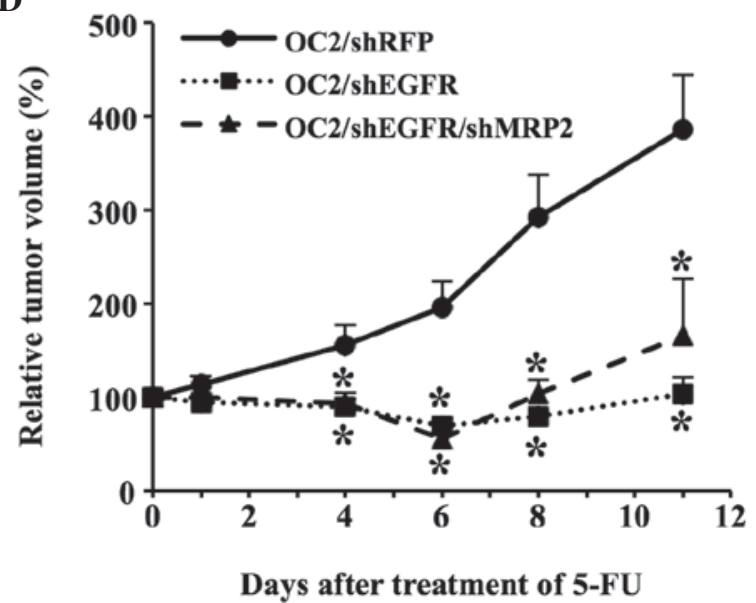

Figure 3. Downregulation of EGFR and $M R P 2$ enhances the sensitivity of OC2 cells to 5-FU. (A) Reduced expression of EGFR and $M R P 2$ in OC2/shEGFR/shMRP2 cells. The messenger RNA levels of EGFR and MRP2 in the cells were quantified using reverse transcription-polymerase chain reaction analysis. The expression levels of EGFR and MRP2 in OC2/shRFP were assigned a value of $100 \%$. (B) Determination of the IC ${ }_{50}$ values of 5-FU in shRNA-transfected OC2 cells. The $\mathrm{IC}_{50}$ value of 5-FU in OC2/shRFP was used as the control. (C) Diagram illustrating the procedure of the in vivo experiments. A total of $5 \times 10^{6}$ shRNA-transfected OC2 cells were subcutaneously implanted into the right dorsal flank of nude mice (n=6/group). When the tumor volumes reached $\sim 100 \mathrm{~mm}^{3}$ (set as day 0 ), the mice were administered intraperitoneal injections of 5 -FU (15 mg/kg) for a total of 6 treatments. The tumor volumes were measured daily. Solid arrow indicates 5-FU treatments on days 0, 1, 2, 7, 8 and 9. (D) In vivo therapeutic effect achieved by the downregulation of EGFR and MRP2. The tumor volumes on day 0 were assigned a value of $100 \%$. The tumor volumes of OC2/shRFP group were used as the controls. Data are presented as the mean \pm standard error. ${ }^{*} \mathrm{P}<0.05$. $^{* *} \mathrm{P}<0.01$. EGFR, epidermal growth factor receptor; MRP2, multidrug resistance-associated protein 2 ; 5-FU, 5-fluorouracil; sh, small hairpin; RFP, red fluorescent protein; $\mathrm{IC}_{50}$, half-maximal inhibitory concentration; s.c., subcutaneously; i.p., intraperitoneal.

the role of MRP2 in OSCC. These cells were generated by treating OC2 cells with cisplatin at progressively increasing concentrations $(0.10,0.25,0.50$ and $0.75 \mu \mathrm{g} / \mathrm{ml})$ until they were able to survive in a medium containing $0.75 \mu \mathrm{g} / \mathrm{ml}$ cisplatin. The expression of EGFR and MRP2 in OC2 and OC2CisR cells was determined using RT-PCR. MRP2 expression was significantly increased in OC2CisR cells, compared with OC2 cells $(\mathrm{P}<0.05)$, whereas $E G F R$ expression did not differ significantly between the two cell lines (Fig. 4A). Cell viability analysis confirmed that OC2CisR cells exhibited an increased resistance to cisplatin, compared with $\mathrm{OC} 2$ cells $(\mathrm{P}<0.05$; Fig. 4B). A cell apoptosis assay additionally confirmed that the OC2CisR cells were more resistant to the killing effect of cisplatin, compared with the OC2 cell line (Fig. 4C).

Furthermore, mice bearing OC2 and OC2CisR subcutaneous tumors were treated with cisplatin to evaluate whether
OC2CisR cells were more resistant to cisplatin, compared with OC2 cells in vivo (Fig. 4D). It was observed that the growth rate of OC2CisR subcutaneous tumors was significantly higher than that of OC2 subcutaneous tumors in the mice following cisplatin administration (Fig. 4E). The tumor growth rate was $52.1 \%$ higher per day in the OC2CisR group, compared with the OC2 group $(\mathrm{SE}=23.0 \% ; \mathrm{P}=0.024)$. These observations confirmed that $\mathrm{OC} 2 \mathrm{CisR}$ cells are more resistant to cisplatin, compared with OC2 cells in vitro and in vivo, suggesting that elevated expression of $M R P 2$ in $\mathrm{OC} 2 \mathrm{C}$ isR cells contributed to their resistance to cisplatin.

Downregulation of EGFR and MRP2 increases the inhibitory effects of cisplatin on the growth of OC2CisR tumors. OC2CisR cells were transfected with shMRP2 and shEGFR to establish whetherMRP2 had a significant role in cisplatin resistance in these cells, and to observe whether downregulation 
A

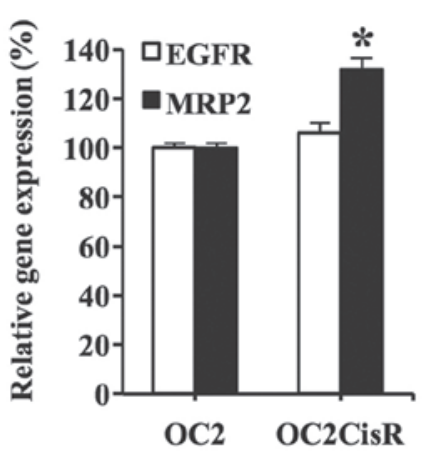

D

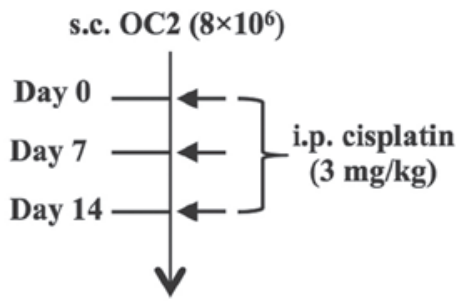

Measurement of tumor volume
B

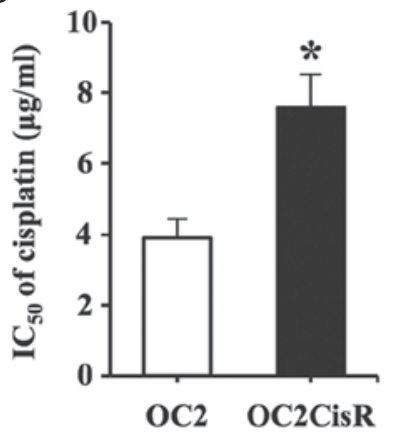

$\mathbf{E}$
C

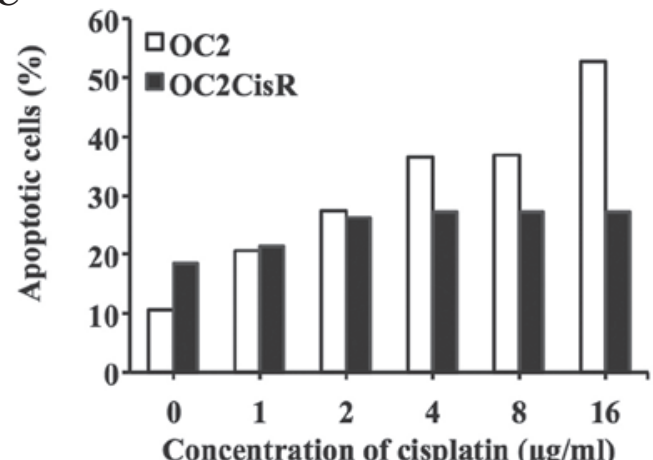

Figure 4. Development of the OC2CisR cell line. (A) Expression of EGFR and $M R P 2$ in OC2CisR cells. The messenger RNA levels of EGFR and $M R P 2$ in $O C 2 C i s R$ cells were quantified using reverse transcription-polymerase chain reaction analysis. The expression levels of $E G F R$ and $M R P 2$ in OC2 cells were assigned a value of $100 \%$ as controls. (B) Determination of the $\mathrm{IC}_{50}$ values of cisplatin in cells. The $\mathrm{IC}_{50}$ value of cisplatin in OC2 was used as the control (C) Cell apoptosis assay. OC2 and OC2CisR cells were exposed to increasing concentrations of cisplatin ranging from 1 to $16 \mu \mathrm{g} / \mathrm{ml}$ for $24 \mathrm{~h}$, and stained with Annexin V-fluorescein isothiocyanate and propidium iodide, followed by flow cytometric analysis. The percentage of total apoptotic cells is shown. (D) Diagram illustrating the procedure of the in vivo experiments. A total of $8 \times 10^{6}$ cells were subcutaneously implanted into the right dorsal flank of nude mice ( $\mathrm{n}=6$ /group). When the tumor volumes reached $\sim 100 \mathrm{~mm}^{3}$ (set as day 0 ), the mice were administered intraperitoneal. injections of cisplatin (3 mg/kg) for a total of 3 treatments. The tumor volumes were measured daily. Solid arrow indicates cisplatin treatment on days 0,7 and 14 . (E) Comparison of tumor growth rates in the mice following cisplatin administration. The tumor volumes on day 0 were assigned a value of $100 \%$. The interaction of time and group in the generalized linear model with generalized estimated equation was $52.1 \%(\mathrm{P}=0.024)$, which indicated that the linear growth rate was $52.1 \%$ higher per day for the OC2CisR group compared with the OC2 group. Data are presented as the mean \pm standard error. * $\mathrm{P}<0.05$. CisR, cisplatin-resistant; EGFR, epidermal growth factor receptor; MRP2, multidrug resistance-associated protein 2; $\mathrm{IC}_{50}$, half-maximal inhibitory concentration; s.c., subcutaneously; i.p., intraperitoneal.
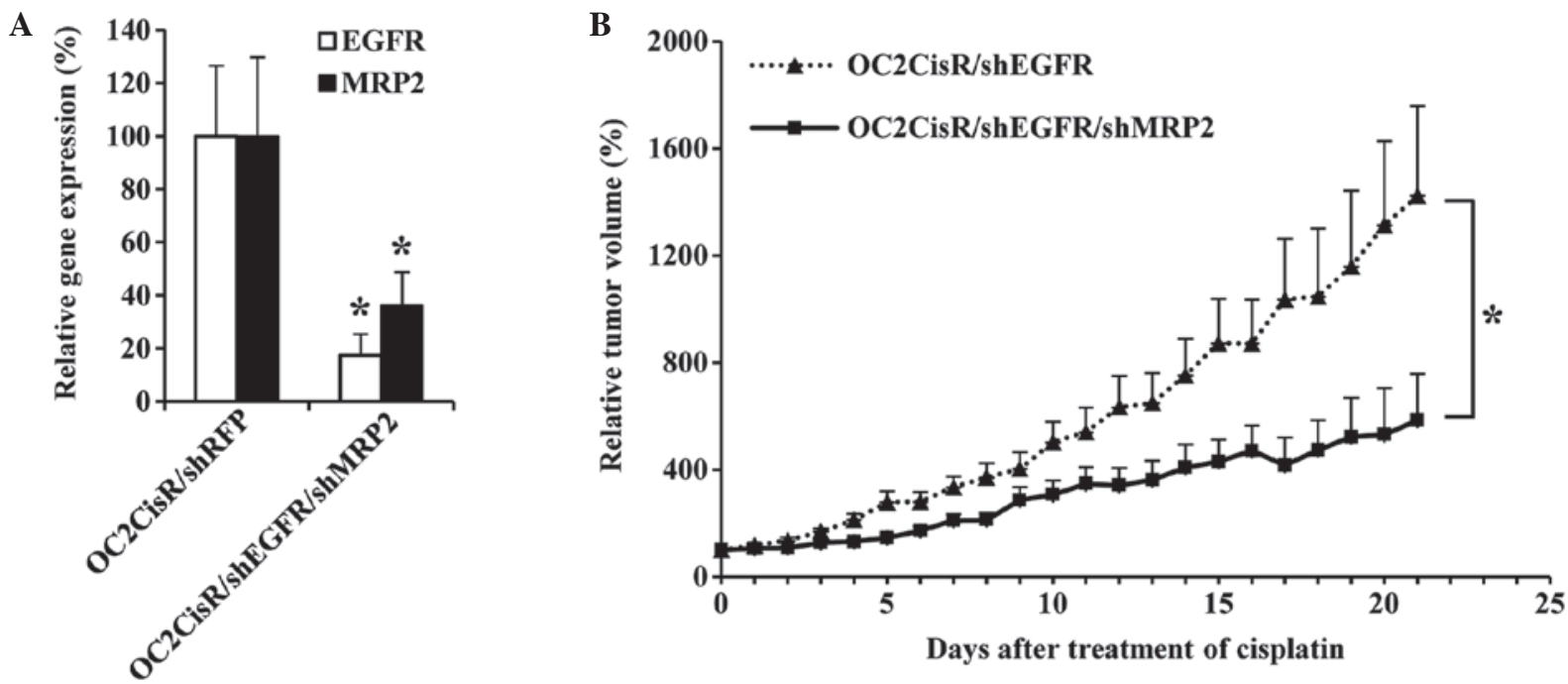

Figure 5. Downregulation of EGFR and $M R P 2$ increases the inhibitory effects of cisplatin on the growth of OC2CisR tumors. (A) Reduced expression of EGFR and $M R P 2$ in OC2cisR/shEGFR/shMRP2 cells. The messenger RNA levels of EGFR and $M R P 2$ in cells was quantified using reverse transcription-polymerase chain reaction analysis. The expression levels of EGFR and $M R P 2$ in OC2/shRFP were assigned a value of $100 \%$ as controls. (B) Therapeutic effect achieved by the downregulation of EGFR and MRP2 in OC2CisR tumors. The procedure of the in vivo experiments is illustrated in Fig. 4D (n=6/group). The tumor volumes on day 0 were assigned a value of $100 \%$. The interaction of time and group in the generalized linear model with generalized estimated equation was $37.4 \%(\mathrm{P}=0.035)$, which indicated that the linear growth rate was $37.4 \%$ higher per day for the OC2CisR/shEGFR group compared with the OC2CisR/shEGFR/shMRP2 group. Data are presented as the mean \pm standard error. ${ }^{*} \mathrm{P}<0.05$. EGFR, epidermal growth factor receptor; MRP2, multidrug resistance-associated protein 2; CisR, cisplatin-resistant; sh, small hairpin; RFP, red fluorescent protein. 
of MRP2 was able to enhance the therapeutic effects of cisplatin in EGFR-downregulated cells. Fig. 5A demonstrates the RNAi-mediated downregulation of EGFR and MRP2 expression in OC2CisR/shEGFR/shMRP2 cells in vitro, compared with the control group OC2CisR/shRFP $(\mathrm{P}<0.05)$. In vivo, the growth rate of OC2CisR/shEGFR/shMRP2 subcutaneous tumors was significantly lower, compared with that of OC2CisR/shEGFR subcutaneous tumors in the mice following cisplatin administration (Fig. 5B). The tumor growth rate was $37.4 \%$ lower per day in the OC2CisR/shEGFR/shMRP2 group, compared with the OC2CisR/shEGFR group ( $\mathrm{SE}=17.8 \% ; \mathrm{P}=0.035)$. These results confirmed that $M R P 2$ overexpression confers resistance to cisplatin in OC2CisR cells, and that downregulation of $M R P 2$ is able to significantly enhance the therapeutic effects of cisplatin in EGFR-downregulated cells.

\section{Discussion}

Overexpression of EGFR is frequently observed in numerous types of cancer, including OSCC $(26,27)$. EGFR-targeted therapies, including gefitinib (Iressa ${ }^{\circledR}$; AstraZeneca, London, UK) $(26,28-30)$ and cetuximab (Erbitux ${ }^{\circledR}$; Eli Lilly and Company, Indianapolis, IN, USA) $(31,32)$, have been studied extensively as potential methods of blocking the cancer-promoting functions of EGFR in OSCC. However, although it has been reported that EGFR inhibitors are initially beneficial for the treatment of cancer, resistance to the inhibitors eventually develops $(21,33)$. Therefore, an alternative strategy was investigated in the present study, in which rather than targeting the EGFR protein, the expression of its gene, $E G F R$, in OSCC was downregulated using RNAi techniques. The results of the present study demonstrate that reduction of EGFR expression inhibits the growth and enhances the cytotoxicity of 5-FU in OSCC cells.

Although MRP2 downregulation also increased the sensitivity of OC2 cells to 5-FU, it did not contribute to the suppression of EGFR-downregulated OC2 tumors following 5-FU administration. Therefore, the ability of $M R P 2$ downregulation to achieve further suppression of $E G F R$-downregulated OC2 tumors in vivo during cisplatin administration was investigated. Following establishment of the cisplatin-resistant cell line OC2CisR, it was observed that the expression of MRP2 was positively correlated with the level of cisplatin resistance in the cells, whereby the OC2CisR cells became highly resistant to the killing effect of cisplatin, compared with the parental cell line OC2. These results imply that MRP2 may have a significant role in modulating the response of OC2CisR cells to cisplatin. Furthermore, downregulation of MRP2 significantly enhanced the therapeutic effect of cisplatin in EGFR-downregulated cells.

In conclusion, MRP2-targeted therapy may be a promising strategy for overcoming the resistance to cisplatin in OSCC. Inhibitors of ABC transporters, including MRP2, have been extensively investigated in previous studies (34). However, there has been little impact of these studies on clinical outcomes $(35,36)$. Therefore, an RNAi-mediated targeting strategy was applied in the present study, with a view to reversing multidrug resistance in tumors. Due to its specificity and effectiveness, RNAi-mediated downregulation of MRP2 may be applicable as a therapeutic approach toward reversing MRP2-dependent cisplatin resistance in OSCC.

\section{Acknowledgements}

The present study was supported by the Ditmanson Medical Foundation Chia-Yi Christian Hospital (Chia-Yi, Taiwan; grant no. R100-3) and the National Science Council of Taiwan (Taipei, Taiwan; grant no. NSC99-2320-B-194-004-MY3).

\section{References}

1. Petersen PE: Strengthening the prevention of oral cancer: The WHO perspective. Community Dent Oral Epidemiol 33: 397-399, 2005.

2. de Camargo Cancela M, Voti L, Guerra-Yi M, Chapuis F, Mazuir M and Curado MP: Oral cavity cancer in developed and in developing countries: Population-based incidence. Head Neck 32: 357-367, 2010.

3. 2009 Statistics of Causes of Death. Ministry of Health and Welfare. Taipei, Taiwan, 2010.

4. Chabner BA and Roberts TG Jr: Timeline: Chemotherapy and the war on cancer. Nat Rev Cancer 5: 65-72, 2005.

5. Andreadis C, Vahtsevanos K, Sidiras T, Thomaidis I, Antoniadis K and Mouratidou D: 5-Fluorouracil and cisplatin in the treatment of advanced oral cancer. Oral Oncol 39: 380-385, 2003.

6. Ramos P and Bentires-Alj M: Mechanism-based cancer therapy: Resistance to therapy, therapy for resistance. Oncogene 34: 3617-3626, 2015.

7. Gottesman MM, Fojo T and Bates SE: Multidrug resistance in cancer: Role of ATP-dependent transporters. Nat Rev Cancer 2: 48-58, 2002.

8. Dean M, Rzhetsky A and Allikmets R: The human ATP-binding cassette (ABC) transporter superfamily. Genome Res 11: 1156-1166, 2001.

9. Stavrovskaya AA and Stromskaya TP: Transport proteins of the $\mathrm{ABC}$ family and multidrug resistance of tumor cells. Biochemistry (Mosc) 73: 592-604, 2008.

10. Surowiak P, Materna V, Kaplenko I, Spaczynski M, Dolinska-Krajewska B, Gebarowska E, Dietel M, Zabel M and Lage H: ABCC2 (MRP2, cMOAT) can be localized in the nuclear membrane of ovarian carcinomas and correlates with resistance to cisplatin and clinical outcome. Clin Cancer Res 12: 7149-7158, 2006

11. Hall MD, Okabe M, Shen DW, Liang XJ and Gottesman MM: The role of cellular accumulation in determining sensitivity to platinum-based chemotherapy. Annu Rev Pharmacol Toxicol 48: 495-535, 2008.

12. Yamasaki M, Makino T, Masuzawa T, Kurokawa Y, Miyata $H$, Takiguchi S, Nakajima K, Fujiwara Y, Matsuura N, Mori M and Doki Y: Role of multidrug resistance protein 2 (MRP2) in chemoresistance and clinical outcome in oesophageal squamous cell carcinoma. Br J Cancer 104: 707-713, 2011.

13. Kool M, de Haas M, Scheffer GL, Scheper RJ, van Eijk MJ, Juijn JA, Baas F and Borst P: Analysis of expression of cMOAT (MRP2), MRP3, MRP4, and MRP5, homologues of the multidrug resistance-associated protein gene (MRP1), in human cancer cell lines. Cancer Res 57: 3537-3547, 1997.

14. Guminski AD, Balleine RL, Chiew YE, Webster LR, Tapner M, Farrell GC, Harnett PR and Defazio A: MRP2 (ABCC2) and cisplatin sensitivity in hepatocytes and human ovarian carcinoma. Gynecol Oncol 100: 239-246, 2006.

15. Taniguchi K, Wada M, Kohno K, Nakamura T, Kawabe T, Kawakami M, Kagotani K, Okumura K, Akiyama S and Kuwano M: A human canalicular multispecific organic anion transporter (cMOAT) gene is overexpressed in cisplatin-resistant human cancer cell lines with decreased drug accumulation. Cancer Res 56: 4124-4129, 1996.

16. Materna V, Stege A, Surowiak P, Priebsch A and Lage H: RNA interference-triggered reversal of ABCC2-dependent cisplatin resistance in human cancer cells. Biochem Biophys Res Commun 348: 153-157, 2006.

17. Xie SM, Fang WY, Liu Z, Wang SX, Li X, Liu TF, Xie WB and Yao KT: Lentivirus-mediated RNAi silencing targeting ABCC2 increasing the sensitivity of a human nasopharyngeal carcinoma cell line against cisplatin. J Transl Med 6: 55, 2008. 
18. Minegaki T, Takara K, Hamaguchi R, Tsujimoto $M$ and Nishiguchi K: Factors affecting the sensitivity of human-derived esophageal carcinoma cell lines to 5-fluorouracil and cisplatin. Oncol Lett 5: 427-434, 2013.

19. Agulnik M: New approaches to EGFR inhibition for locally advanced or metastatic squamous cell carcinoma of the head and neck (SCCHN). Med Oncol 29: 2481-2491, 2012.

20. Mendelsohn J and Baselga J: Epidermal growth factor receptor targeting in cancer. Semin Oncol 33: 369-385, 2006.

21. Chong CR and Jänne PA: The quest to overcome resistance to EGFR-targeted therapies in cancer. Nat Med 19: 1389-1400, 2013.

22. Zhang M, Zhang X, Bai CX, Song XR, Chen J, Gao L, Hu J, Hong QY, West MJ and Wei MQ: Silencing the epidermal growth factor receptor gene with RNAi may be developed as a potential therapy for non small cell lung cancer. Genet Vaccines Ther 3: 5,2005

23. Wong DY, Chang KW, Chen CF and Chang RC: Characterization of two new cell lines derived from oral cavity human squamous cell carcinomas - OC1 and OC2. J Oral Maxillofac Surg 48: 385-390, 1990

24. Lai YH, He RY, Chou JL, Chan MW, Li YF and Tai CK: Promoter hypermethylation and silencing of tissue factor pathway inhibitor-2 in oral squamous cell carcinoma. J Transl Med 12: 237, 2014.

25. Shichinohe $T$, Bochner BH, Mizutani K, Nishida $M$, Hegerich-Gilliam S, Naldini L and Kasahara N: Development of lentiviral vectors for antiangiogenic gene delivery. Cancer Gene Ther 8: 879-889, 2001.

26. Shintani S, Li C, Mihara M, Nakashiro K and Hamakawa H: Gefitinib ('Iressa'), an epidermal growth factor receptor tyrosine kinase inhibitor, mediates the inhibition of lymph node metastasis in oral cancer cells. Cancer Lett 201: 149-155, 2003.

27. Takaoka S, Iwase M, Uchida M, Yoshiba S, Kondo G, Watanabe H, Ohashi M, Nagumo M and Shintani S: Effect of combining epidermal growth factor receptor inhibitors and cisplatin on proliferation and apoptosis of oral squamous cell carcinoma cells. Int J Oncol 30: 1469-1476, 2007.
28. Shintani S, Li C, Mihara M, Terakado N, Yano J, Nakashiro K and Hamakawa H: Enhancement of tumor radioresponse by combined treatment with gefitinib (Iressa, ZD1839), an epidermal growth factor receptor tyrosine kinase inhibitor, is accompanied by inhibition of DNA damage repair and cell growth in oral cancer. Int J Cancer 107: 1030-1037, 2003.

29. Shintani S, Li C, Mihara M, Yano J, Terakado N, Nakashiro K and Hamakawa H: Gefitinib ('Iressa', ZD1839), an epidermal growth factor receptor tyrosine kinase inhibitor, up-regulates p27KIP1 and induces G1 arrest in oral squamous cell carcinoma cell lines. Oral Oncol 40: 43-51, 2004.

30. Lee EJ, Whang JH, Jeon NK and Kim J: The epidermal growth factor receptor tyrosine kinase inhibitor ZD1839 (Iressa) suppresses proliferation and invasion of human oral squamous carcinoma cells via p53 independent and MMP, uPAR dependent mechanism. Ann N Y Acad Sci 1095: 113-128, 2007.

31. Dai W, Li Y, Zhou Q, Xu Z, Sun C, Tan X and Lu L: Cetuximab inhibits oral squamous cell carcinoma invasion and metastasis via degradation of epidermal growth factor receptor. J Oral Pathol Med 43: 250-257, 2014.

32. Qian M, Qian D, Jing H, Li Y, Ma C and Zhou Y: Combined cetuximab and celecoxib treatment exhibits a synergistic anticancer effect on human oral squamous cell carcinoma in vitro and in vivo. Oncol Rep 32: 1681-1688, 2014.

33. Ohnishi Y, Minamino Y, Kakudo K and Nozaki M: Resistance of oral squamous cell carcinoma cells to cetuximab is associated with EGFR insensitivity and enhanced stem cell-like potency. Oncol Rep 32: 780-786, 2014

34. Zhou SF, Wang LL, Di YM, Xue CC, Duan W, Li CG and Li Y Substrates and inhibitors of human multidrug resistance associated proteins and the implications in drug development. Curr Med Chem 15: 1981-2039, 2008.

35. Falasca $M$ and Linton KJ: Investigational ABC transporter inhibitors. Expert Opin Investig Drugs 21: 657-666, 2012.

36. Yu M, Ocana A and Tannock IF: Reversal of ATP-binding cassette drug transporter activity to modulate chemoresistance: Why has it failed to provide clinical benefit? Cancer Metastasis Rev 32: 211-227, 2013 